\title{
Relationship of the Traditional Chinese Medicine Constitution and Skin Moist Degree
}

Hong Meng, Yifan He, Xiaoyang Zheng, Wenyi Zhu, Yinmao Dong”

China Cosmetic Collaborative Innovation Center, Beijing Technology and Business University, Beijing, China

"Corresponding author: Yinmao Dong, China Cosmetic Collaborative Innovation Center, Beijing Technology and Business University, No. 11/33, Fucheng Road, Haidian District, Beijing, China Tel.: +861068984937 Fax: +861068984937 Email: ymdong2008@163.com

Received May 16, 2016

Revised July 14, 2016

Accepted July 22, 2016

Published September 30, 2016

\begin{abstract}
Purpose: We aim to study the relationship between the traditional Chinese medicine constitutional types and skin moisture degree which includes water content, loss of water, and oil content, for permanent population in Beijing. Methods: 246 women are randomly selected as volunteers and are classified according to Wang's nine physical inventory methods. We detected the amount of moisture, water loss, and oil content for the volunteers (forehead, left canthus, left cheek, and chin). The data obtained from relative large number of mild constitution, yin deficiency, yang deficiency, phlegm-wet, damp-heat, qi deficiency, and qi depression are compared and analyzed. Results: Forehead and eyelid water content of phlegm-wet is higher than other groups. In addition, left cheek and chin water content of yang deficiency type is higher than others. Moreover, oil content of phlegm-wet is higher than other groups. Conclusion: Constitution is the important factor that affects the skin moist degree, and is closely relevant with skin moisture content and oil content.
\end{abstract}

Keywords: Traditional Chinese medicine constitutional types, Skin moist degree, Water content, Transepidermal water loss, Oil content

\section{Introduction}

体质是特定躯体素质与一定心理素质的综合体，是 “形神合一” 思想在中医体质学说中的体现 (Wang, 2002)。皮肤是人体最大的器官, 也是人体第一道屏 障，起着防体内的营养物质流失、水分等的流失，维持 皮肤的含水量, 保持肌肤的滋润等作用 ( He, 2011)。 滋润度标志着皮肤的代谢功能，展示皮肤细淢、柔嫩、 光滑和富有弹性的特征, 当皮肤屏障受到破坏, 经表皮 水分散失增加, 皮肤就会变得干燥 (He, 2011)。汗液与 皮脂的相互乳化后产生的产物, 可使皮肤柔软, 润泽, 防止干裂等作用。所以皮肤的滋润度是决定着皮肤的 健康程度重要因素。而中医讲“有其内而必形于外”, 如《外科正宗》: “气血不荣, 皮肤枯槁, 手足皮枯 槁…… 、《内经·素问》: “汗出见湿, 乃生痤疿”, 这 两种分别代表着不同的体质对皮肤产生的不同影响。所 以，体质也是影响皮肤健康程度的一个重要因素。体质
因素是皮肤类型和表征的重要物质基础，每种体质类型 都有特定的皮肤特征。本文以水分含量、水分散失量、油脂 含量为测试指标研究中医体质与皮肤的滋润度的相关性。

\section{Methods}

\section{Objects of study}

246名女性参加试验, 年龄21-49岁, 平均 (27.31 10.04) 岁。数据采集时间为2015年3月8日-2015年12月8日， 所有受试者面部皮肤外观健康光滑, 无红斑、脱屑等皮 疹, 无其他皮肤疾患或系统性疾病。试验前, 每位志愿 者被告知试验的过程, 并签署知情同意书。然后按王琦 教授九种体质分类 (Wang, 2005) 受试者进行体质分型, 因 血瘀质和特禀质人数较少, 为避免对比数据误差过大, 故 选取人数相对较多的平和质37人、阳虚质37人、阴虚质35人、 㾳湿质 18 人、湿热质 19 人、气虚质44人、气郁质32人的测试结 
果进行面部 4 个位点（额头、左眼角、左脸规和下巴），3个测试 项目的对比。

\section{Test instruments}

水分含量用水分测试仪Cormeometer (CM825; Courage +Khazaka electronic GmbH， Germany），采用电容原 理, 可以测量参数: 湿度测量值 (moisture measurement value, MMV)，MMV越大，表示皮肤水分含量越大。

水分散失量用皮肤水分流失 transepidermal water loss (TEWL)测试仪Tewamater (TM300; Courage+Khazaka electronic $\mathrm{GmbH}$, Germany) 测量, 原理是菲克扩散定 律, 可以测量参数, 皮肤保护层越完好, 水分的含量就会越 高, 皮肤水分流失TEWL的数值就越低, TEWL的单位为 $\mathrm{g} / \mathrm{hm}^{2}$ 。 皮肤油脂测试仪Sebumeter (SM815; Courage+Khazaka electronic $\mathrm{GmbH}$, Germany), 利用光度测定法, 可以测量 参数: 油脂含量, 单位为 $\mu \mathrm{g} / \mathrm{cm}^{2}$ 。

\section{Detection methods}

1) Skin water content

选择屏幕中 “Measurement” 对应按钮 “1”键进入测试界 面。将水分测试探头垂直地轻压在被测皮肤表面, 听到提 示音测试完成。屏幕显示出测试结果, 每个部位重复测试5 次。记录最后一行平均值数据, 即该处的平均水分含量。

\section{2) Transepidermal water loss}

选择屏幕中 “Measurement” 对应按钮 “1”键进入测试 界面。将水分流失测试探头平的一端轻放在被测皮肤表 面, 按探头手柄上的按钮 (或者选择屏幕上 “START” 对 应的按钮 “2”键) 开始测试。测试时间为20s, 按探头手 柄上的按钮 (或者选择屏幕上 “STOP” 对应的按钮“2”
键) 停止测试, 记录第二行数据, 即在规定时间内, 该处皮 肤平均水分散失量。

3) Oil content

将油脂含量测试盒轻按抵住槽底一秒, 听到提示音后测试 开始, 屏幕上显示测试倒计时。将油脂含量测试盒轻按于 测试部位, 保持稳定。测试时间一般为 $20 \mathrm{~s}$, 倒计时结束 后, 将油脂含量测试盒放进槽内, 轻按抵住槽底一秒, 屏 幕会显示测量值。观察屏幕上的数字，上面 “1、2、3、4” 四行分别对应四次测试的结果, 最下面一行为平均值。

\section{Test environment}

选择 $20^{\circ} \mathrm{C}$ 和 $50 \%$ 相对湿度环境下测量。

\section{Statistical analysis}

选用 SPSS 22.0 数据处理系统, 采用单因素方差分析比较皮 肤水分含量、水分散失量、油脂含量在不同体质的差异性。

\section{Results and Discussion}

\section{The relationship of the traditional Chinese medicine constitution and moisture content}

从Table 1中痰湿质和阳虚质的水分含量高于其他5组, 即 瘳湿质的额头、眼角的水分含量均高于其他几组的水分含 量。阳虚质的左脸规和下领水分含量数值高于其他几组; 具体来说与平和质组相比除气虚质、湿热质下领比平和质 下领的水分含量略低外, 其他几组测试部位的水分含量均 高于平和质测试部位的水分含量, 其中, 湿热质左眼角最 为明显 $(p<.01)$; 与阳虚质相比, 湿热质左眼角水分含量与

Table 1. Seven physical average moisture content parameters from different parts of the face $(X \pm S)$

\begin{tabular}{lcclcc}
\hline & Total N=222 & Forehead & Left canthus & Left cheek & Chin \\
\hline Mild constitution & 37 & $46.96 \pm 14.61$ & $60.47 \pm 13.86^{\text {光方 }}$ & $42.51 \pm 16.17$ & $45.53 \pm 10.97$ \\
Yang deficiency & 37 & $54.34 \pm 14.71$ & $64.97 \pm 17$ & $54.05 \pm 16.66$ & $60.72 \pm 51.99$ \\
Yin deficiency & 35 & $51.97 \pm 12.44$ & $63.59 \pm 13.71$ & $44.74 \pm 13.81$ & $47.55 \pm 11.88$ \\
Phlegm-wet & 18 & $61.21 \pm 14.20$ & $67.17 \pm 13.56$ & $53.40 \pm 11.26$ & $54.03 \pm 10.99$ \\
Damp-heat & 19 & $50.97 \pm 12.52$ & $60.49 \pm 13.63^{*} \triangle$ & $49.32 \pm 13.18$ & $40.65 \pm 14.30$ \\
Qi deficiency & 44 & $52.81 \pm 13.91$ & $62.34 \pm 12.27$ & $51.49 \pm 13.34$ & $42.65 \pm 14.69$ \\
Qi depression & 32 & $50.68 \pm 12.84$ & $62.42 \pm 13.23$ & $46.65 \pm 14.78$ & $47.28 \pm 12.54$ \\
\hline
\end{tabular}

Comparison between groups, ${ }^{*}$ Statistical differences compared with mild constitution and significant differences ${ }^{*} p<.05,{ }^{* *} p<.01$;

${ }^{\triangle}$ Statistical differences compared with yang deficiency and significant differences $\triangle_{p}<.05,{ }^{\Delta} p<.01$;

Statistical differences compared with yin deficiency and significant differences $p<.05$, th $p<.01$;

"Statistical differences compared with damp-heat and significant differences ${ }^{\#} p<.05$, ${ }^{\# \#} p<.01$;

${ }^{0}$ Statistical differences compared with qi deficiency and significant differences ${ }^{0} p<.05,{ }^{00} p<.01$;

^ Statistical differences compared with qi depression and significant differences $\hat{p}<.05,{ }^{\wedge} p<.01$. 
之相近 $(p<.05)$; 与阴虚质相比，平和质左眼角水分含量与 之相近 $(p<.01)$ 。

\section{The relationship of the traditional Chinese medicine} constitution and transepidermal water loss

从Table 2中可以看出痰湿质的额头水分散失量平均值高于 其他几组; 气郁质左眼角水分散失量平均值高于其他组, 气 虚质左脸颊、下领水分散失量平均值高于其他组。

\section{Relationship of traditional Chinese medicine constitution and oil content}

从Table 3中可以看出痰湿质测试部位的油脂含量均高于其 他几组测试部位油脂含量。具体来说与平和质相比, 湿热 质左眼角含量低于平和质左眼角 $(p<.05)$, 气虚质下领油脂 含量低于平和质下领油脂含量 $(p<.01)$; 气虚质、气郁质左 脸颊、痰湿质下领油脂含量高于平和质左脸颊 $(p<.05)$ 、下 领油脂含量 $(p<.01)$; 与阳虚质相比，平和质左脸规油脂含 量低于阳虚质油脂含量 $(p<.01)$; 与阴虚质相比，阳虚质 额头、湿热质和气郁质左脸规的油脂含量均低于阴虚质测 试部位的油脂含量 $(p<.05)$; 与湿热质相比，气虚质额头
油脂含量与之相近 $(p<.01)$; 与气虚质相比, 平和质、气 郁质额头油脂含量高于气虚质额头油脂含量 $(p<.05)$, 而阳 虚质额头油脂含量与之相近 $(p<.05)$; 与气郁质相比，湿热 质左眼角油脂含量低于气郁质左眼角油脂含量 $(p<.01)$, 平 和质左脸项油脂含量低于气郁质左脸项油脂含量 $(p<.05)$ 。

\section{Conclusion}

王琦教授总结九种体质的皮肤特点：平和质人群面部多红 润有光泽、基本处于正常状态; 气虚质人群面色偏黄或㿠白 且皮肤松弛; 阳虚质人群面色柔白, 目胞晦暗; 阴虚质人群 面部易干且易生皱纹, 色苍或潮红; 痰湿质人群面多油脂, 面色淡黄而暗, 眼胞微浮; 湿热质人群面垢油光, 易生痤疮 粉刺等; 气郁质人群皮肤多干燥，面色、毛发无光泽。从上 面Table 1和Table 3中可以看到相对的平和的平和质水分含量 并不是最高, 结果与之前文献的调研相符 (Li et al., 2012), 痰湿质的水分含量及额头油脂含量相对最高, 痰湿质体征 及皮肤特征因水湿内停而痰湿凝聚, 上泛肌肤而导致体形肥 胖, 面多油脂, 眼胞微浮 (Wang et al., 2006) 等; 另有文献记

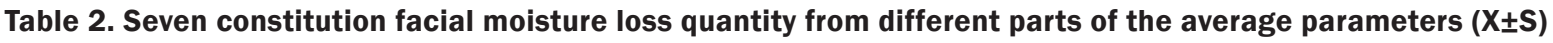

\begin{tabular}{lccccc}
\hline & Total N=222 & Forehead & Left canthus & Left cheek & Chin \\
\hline Mild constitution & 37 & $24.2 \pm 7.75$ & $23.61 \pm 7.74$ & $24.50 \pm 9.55$ & $24.99 \pm 9.11$ \\
Yang deficiency & 37 & $23.00 \pm 8.82$ & $22.61 \pm 8.52$ & $21.49 \pm 6.72$ & $23.32 \pm 9.68$ \\
Yin deficiency & 35 & $24.49 \pm 10.78$ & $21.93 \pm 8.74$ & $22.94 \pm 9.73$ & $21.45 \pm 12.55$ \\
Phlegm-wet & 18 & $26.01 \pm 11.34$ & $21.50 \pm 5.68$ & $22.78 \pm 7.46$ & $24.53 \pm 13.31$ \\
Damp-heat & 19 & $21.61 \pm 5.01$ & $19.09 \pm 6.16$ & $21.48 \pm 7.46$ & $24.53 \pm 13.49$ \\
Qi deficiency & 44 & $25.96 \pm 16.86$ & $22.84 \pm 13.76$ & $23.17 \pm 11.42$ & $28.15 \pm 14.31$ \\
Qi depression & 32 & $23.00 \pm 6.17$ & $23.95 \pm 11.84$ & $21.55 \pm 7.78$ & $26.52 \pm 13.26$ \\
\hline
\end{tabular}

Table 3. Seven constitution facial grease content from different parts of the average parameters $(X \pm S)$

\begin{tabular}{|c|c|c|c|c|c|}
\hline & Total $\mathrm{N}=222$ & Forehead & Left canthus & Left cheek & Chin \\
\hline Mild constitution & 37 & $32.80 \pm 31.07^{0}$ & $13.49 \pm 15.73^{\#^{\wedge}}$ & $14.49 \pm 13.87^{\Delta^{\wedge}}$ & $27.65 \pm 22.33$ \\
\hline Yang deficiency & 37 & $27.31 \pm 18.86$ & $14.57 \pm 17.54$ & $15.17 \pm 15.38$ & $28.89 \pm 23.61$ \\
\hline Yin deficiency & 35 & $29.48 \pm 16.52^{\wedge}$ & $11.24 \pm 9.81$ & $16.67 \pm 14.07$ & $30.15 \pm 19.25$ \\
\hline Phlegm-wet & 18 & $35.24 \pm 16.52$ & $16.12 \pm 10.81$ & $24.59 \pm 17.06$ & $29.83 \pm 21.15^{* \star}$ \\
\hline Damp-heat & 19 & $27.70 \pm 20.37$ & $10.04 \pm 11.42^{\star \wedge \wedge}$ & $12.63 \pm 10.94$ & $23.52 \pm 22.48^{\wedge}$ \\
\hline Qi deficiency & 44 & $27.25 \pm 22.26^{\# \#}$ & $12.10 \pm 15.14$ & $16.19 \pm 16.42^{*}$ & $22.63 \pm 24.91$ * \\
\hline Qi depression & 32 & $30.43 \pm 20.68^{\circ}$ & $14.73 \pm 19.36$ & $19.80 \pm 17.21^{\text {*底 }}$ & $22.97 \pm 17.35$ \\
\hline
\end{tabular}

Comparison between groups, ${ }^{*}$ Statistical differences compared with mild constitution and significant differences ${ }^{*} p<.05,{ }^{* *} p<.01$;

${ }^{\triangle}$ Statistical differences compared with yang deficiency and significant differences ${ }^{\triangle} p<.05, \triangle \Delta_{p}<.01$;

Statistical differences compared with yin deficiency and significant differences $p<.05$, $p<.01$;

${ }^{\#}$ Statistical differences compared with damp-heat and significant differences ${ }^{\#} p<.05,{ }^{\# \#} p<.01$;

${ }^{0}$ Statistical differences compared with qi deficiency and significant differences ${ }^{0} p<.05,{ }^{00} p<.01$;

Statistical differences compared with qi depression and significant differences $\hat{p}<.05,{ }^{\wedge} p<.01$. 
载 “肥胖的痰湿体质的女性可有雄激素增加, 约为正常人2 倍” (Wang, 2011) ; 从皮肤生理学角度, 人体内的雄激素可 影响皮脂腺生长和产生增加, 分泌功能增强 (quoted in Zhu, 2014; He, 2011)，故而在本次测试中见痰湿质组中油脂含量 在皮肤测试中含量最高。皮肤汗腺分泌的水分和皮脂腺分 泌的产物 (包括油脂) 在皮肤表面形成水脂膜, 具有一定的 锁水功能 (He, 2011)。在本次测试中痰湿质在皮肤测试中水 分含量也相对最高, 这与皮肤油脂分泌量有一定的关系。 这类体质的人群护肤时, 建议选择以凝胶啫喱、乳液、润 肤露、保湿精华等保湿清爽的护肤品 (Meng et al., 2012) 以 及做好防晒工作以免因洗护不当或日晒加重油脂分泌过 度 (Chen \& Zhao, 2013) 而导致面部痤疮的发生或加重 (Wu, 2003)。阳虚质是以阳气不足、体内虚寒为主要特征 (Yao \& Wang, 2008), 常常引起机体气化不利, 津液代谢异常, 水液内停, 阻滞于腠理 (Wang et al., 2006)。腠理, 泛指皮 肤、肌肉、脏腑的纹理及皮肤肌肉间隙交接处的结缔组织 (Li, 2001)。津液是体内水液的总称 (He \& Su, 2012)。在皮 肤生理学中皮肤中的分泌的汗液和皮肤中的水分以及溶解 于其中的丰富的“精微物质” (Sun, 2001) 和电解质等属于 “津液”的一部分 (Jian, 2007)，因 “气化不利” 而水滞内 停于腠理，就是说皮肤中水分“内停于腠理” 时，也会导 致体液中的电解质紊乱 (Hou, 2014)，测试皮肤水分含量的 原理是基于水和其他物质的介电常数差异显著, 测得的皮 肤的电容值不同，其观测参数代表皮肤水分值 (Conti et al., 1995; Zuang et al., 1997) ; 故见阳虚质的部分测试部位 (左 脸颊和下领) 的水分含量较高而未见油脂含量高。气郁质眼 角和气虚质左脸项水分散失量均高于其他几组, 而这两种 体质的肌肤特征分别为气虚固摄无力、肌肤失于濡养，气 虚导致卫气的调控功能失常, 卫气主腠理玄府 (汗孔) 的开 合 (Yin, 1984)，若卫气不充，腠理津液渗泻无度而导致汗出 较多、皮肤干燥、细纹、粗糙; 气郁质常常因脏腑功能失 调导致气血运行不畅、津液敷布障碍，不达肌表或郁久化 热, 导致津液外泄而致皮肤干燥、皮肤松弛、皱纹增多。 在评价皮肤屏障功能指标中，皮肤的水分散失量是对皮肤 表面水分蒸发的浓度梯度进行测量的结果。皮肤表面的水 分蒸发多数依靠汗腺的分泌汗液。水分散失量越多皮肤则 易产生细纹，使皮肤干燥、衰老。符合这两种体质测试部 位水分散失量高的表现。这类体质的人群建议在平常护肤 时选择保湿抗衰、增加皮肤胶原蛋白和弹性蛋白成分的营 养霜或乳液 (Meng et al., 2012)；本文根据皮肤滋润度的各 项指标和中医体质进行研究，表明中医体质是影响皮肤滋 润度指标的重要因素。

中医整体观认为, 人是一个统一的整体。皮肤和体质是局部 与整体, 内在与外在的关系体现 (Qu et al., 2014), 结合中医
理论，根据个人体质，因人制宜、因人施美，为我们的 “面 子” 寻找合理的护肤之道。

\section{References}

Chen M, Zhao Y. Oily skin and control of the sebaceous glands. Detergent \& Cosmetics, 36: 32-34, 2013.

Conti A, Schiavi ME, Seidenari S. Capacitance, transepidermal water loss and causal level of sebum in healthy subjects in relation to site, sex and age. International Journal of Cosmetic Science, 17: 77-85, 1995.

He L. Cosmetic dermatology, 2nd edition. People's Medical Publishing House, Beijing, pp34-43, 2011.

He J, Su Y. Handouts of inner canon. People's Medical Publishing House, Beijing, p92, 2012.

Hou SL. Clinical nursing analysis water electrolyte and acidbase imbalances. Medical Frontier, 10: 306-307, 2014.

Zhu XJ. Dermatology. Beijing Medical University Press, Beijing, p619, 2014. (Jean LB, Joseph L, Ronald PR. 2008).

Jian SH. The unity of opposites in the regulation of water, electrolyte metabolism and acid-base balance. China Practical Medicine, 2: 50-52, 2007.

Li JM, Wang X, Qi YH, Zhang N, Li YW, Guo LY. TCM constitution relation with skin moisture and $\mathrm{pH}$ value of research. Acta Chinese Medicine and Pharmacology, 40: 81-82, 2012.

Li JW. Concise dictionary of traditional Chinese medicine. China Press of Traditional Chinese Medicine, Beijing, p1046, 2001.

Meng $\mathrm{H}$, Huang YG, Dong YM. Physique raise a new era. Chinese Medicine Press of China, Beijing, pp52-111, 2012.

Qu JN, Wang J, Xiong WG, Fang C, Dai J, Sun SX, Wang Q, Wei SM. A relevant research on facial skin conditions of Chinese urban females and TCM constitutional types. Chinese Journal of Information on Traditional Chinese Medicine, 10: 22-25, 2014.

Sun LJ. Study on anti-disease action of body fluid and its immunological basis. Shaanxi Journal of Traditional Chinese Medicine, 22: 740-742, 2001. 
Wang Q. Classification and diagnosis basis of nine basic constitutions in Chinese medicine. Journal of Beijing University of Traditional Chinese Medicine, 28: 1-8, 2005.

Wang Q. Statues and prospect of constitutional theory in traditional Chinese medicine. Chinese Journal of Basic Medicine in Traditional Chinese Medicine, 8: 6-15, 2002.

Wang Q. The discovery of nine kinds of Chinese constitution. Science press, Beijing, pp136-169, 2011.

Wang Q, Ye JN, Zhu YB, Gao JH, Dong J, Wang DP, Wu HD. Research on diagnosis standard of phlegm-dampness constitution of TCM. Journal of Chinese Medicine, 21: 73-75, 2006.
Wu XH. Acne pathogenesis research status of Chinese and western medicine. Combine Traditional Chinese and Western Medicine Skin Venereology Magazine in China, 2: 193-197, 2003.

Yao SL, Wang Q. Discussion on the cause of yang-qi deficiency constitution. Chinese Journal of Basic Medicine in Traditional Chinese Medicine, 14: 405407, 2008.

Yin HH. Basic theory of traditional Chinese medicine. Shanghai Science and Technology Publishing House, Shanghai, p58, 1984.

Zuang V, Rona C, Distante F, Berardesca E. The use of a capacitance, device to evaluate the hydration of human skin. Journal of Applied Cosmetology, 15: 95102, 1997. 


\section{中文摘要}

\section{中医体质与皮肤泫润度的关系}

孟宏, 何一凡，郑晓阳，朱文驿，董银卯”

北京工商大学 中国化妆品协同创新中心, 北京, 中国

目的: 研究北京地区常住人群的中医体质与皮肤茲润度 (水分含量、水分散失量、油脂含量)的相关关系。方法: 随机 选取北京地区246名女性志愿者，根据王琦的九种体质分类方法进行体质分类并检测面部 (额头、左眼角、左脸规、下领) 的水分含量、水分散失量、油脂含量, 选取人数相对较多的平和质、阴虚质、阳虚质、痰湿质、湿热质、气虚质、气 郁质的测试数据进行对比。结果：痰湿质的额头、眼角的水分含量均高于其他几组的水分含量。阳虚质的左脸颊和下 领水分含量数值高于其他几组 ; 痰湿质测试部位的油脂含量均高于其他几组测试部位油脂含量。结论: 体质是影响皮 肤泫润度指标的重要因素，体质与皮肤水分含量、油脂含量存在一定关联性。

关键词: 中医体质，皮肤泫润度，水分含量，水分散失量，油脂含量 


\section{국문초록}

\section{중의학 체질과 피부 습윤도의 관계}

맹홍, 하일범, 정효양, 주문이, 동은묘

북경공상대학 중국화장품공동혁신센터, 북경, 중국

목적: 북경지역에 거주하는 거주민을 대상으로 중의학 체질과 피부 습윤도(수분함량, 경피수분손실량, 피지함량)의 상관관 계 연구를 진행하였다. 방법: 북경지역에 거주하는 거주민 중 여성 246 명을 무작위로 피험자로 선발하였다. 그리고 Wangqi 의 9종류 체질분류 방법에 따라 피험자를 분류하고, 각 피험자의 이마, 왼쪽 눈가, 왼쪽 볼, 아래 턱 부위별로 수분함량, 경 피수분손실량, 피지함량을 측정하였다. 그리고 상대적으로 인원수가 많은 평화체질, 음허체질, 양허체질, 담습체질, 습열체 질, 기허체질, 기울체질 등 여러 체질에서 얻은 데이터를 비교 분석하였다. 결과: 담습체질의 이마, 눈가 수분함량은 모두 다 른 체질의 수분함량보다 높고, 양허체질의 왼쪽 볼과 아래 턱의 수분함량 수치는 다른 체질보다 높게 측정되었다. 담습체질 의 측정부위의 피지함량은 모두 다른 체질보다 높게 측정되었다. 결론: 체질은 피부 습윤도 지표에 영향을 주는 중요한 요소 이며 체질과 피부의 수분함량, 경피수분손실량, 피지함량은 일정한 상관관계가 있다고 사료된다.

핵심어: 중의학 체질, 피부 습윤도, 수분함량, 경피수분손실량, 피지함량 
